\title{
Scattering Matrix and Doubling Equations for the Scattering and Transmission Functions ${ }^{1}$
}

\author{
P. T. Y. POON ANd S. UENo \\ Dept. of Electrical Engineering, University of Southern California, Los Angeles 90007
}

15 November 1972 and 29 January 1973

\begin{abstract}
In a series of papers given by Redheffer, Peebles and Plesset, Wang, Aronson and Yarmush, Grant and Hunt, the scattering matrix method has been applied to the solution of the linearized Boltzmann equation. On the other hand, the doubling equations for the scattering and transmission functions in a homogeneous anisotropically scattered atmosphere have been found by van de Hulst and successfully extended by Hansen and Hansen and by Hovenier with the aid of the invariance principles. In the present paper we show how to derive the doubling equations for the scattering and transmission functions in a homogeneous anisotropically scattering atmosphere by using the scattering matrix.
\end{abstract}

\section{Introduction}

It is well known that in the theory of line formation in terrestrial, planetary and stellar atmospheres the solution of the transfer equation plays an important role (cf., Chandrasekhar, 1960; Ambarzumian, 1958; Sobolev, 1962; Busbridge, 1960; Bellman et al, 1963). For this purpose we use the order-of-scattering theory of radiation, whose solution converges to the solution of multiple scattering processes in the limit as the number of collisions tends to infinity. The required solutions are given either by the successive approximation or by the doubling (or adding) equations. In a series of papers given by several authors (cf., van de Hulst, 1963; Uesugi and Irvine, 1969; Hovenier, 1971; Bellman et al., 1972), the former solutions have been discussed. On the other hand, the doubling method has been successfully developed and applied to the planetary atmospheric problems (cf., van de Hulst, 1963; Hansen, 1969; Hansen and Hovenier, 1971; Ueno and Wang, 1973). Here, with the aid of the scattering matrix (cf., Redheffer, 1962; Peebles and Plesset, 1951; Wang, 1966; Aronson and Yarmush, 1966; Grant and Hunt, 1969), we show how to find the doubling equations for the scattering and transmission functions for a homogeneous, isotropically scattering atmosphere. In a subsequent paper we shall adopt the scattering matrix approach to the solution of the transfer equation for Chandrasekhar's stratified planetary problem.

\footnotetext{
' Supported by the National Science Foundation under Grant GP 29049 and the Atomic Energy Commission, Division of Research, under Contract AT(40-3)-113, Project 19.
}

\section{Scattering matrices and the star product}

Consider a plane-parallel, inhomogeneous, anisotropically scattering atmosphere, whose top at the optical depth $x$ and bottom at $z(0 \leqslant x \leqslant z)$ are together monodirectionally illuminated and are bounded by nonreentrant surfaces. The angles of incidence on the boundaries at the upper and lower surfaces are denoted by $\boldsymbol{\Omega}_{x}=\left(-u_{x}, \phi_{x}\right) \quad\left(0<u_{x} \leqslant 1, \quad 0 \leqslant \phi_{x} \leqslant 2 \pi\right)$ and $\Omega_{z}$ $=\left(u_{z}, \phi_{z}\right)\left(0<u_{z} \leqslant 1,0 \leqslant \phi_{z}<2 \pi\right)$, where $u_{x}$ (or $\left.u_{z}\right)$ represents the cosine of inclination to the downward directed normal and $\phi_{x}$ (or $\phi_{z}$ ) the azimuthal angle.

Let the upwelling intensity of radiation at level $t(x \leqslant t \leqslant z)$ in the direction $\Omega=(v, \phi)(0 \leqslant v \leqslant 1,0 \leqslant \phi$ $\leqslant 2 \pi)$ be denoted by $\mathbb{I}(l, \Omega)$, and similarly let the downwelling intensity of radiation at level $l$ in the direction $-\boldsymbol{\Omega}=(-v, \phi)(0 \leqslant v \leqslant 1,0 \leqslant \phi \leqslant 2 \pi)$ be denoted by $\mathbf{I}(t,-\Omega)$, where $v$ is the cosine of inclination to the upward normal and $\phi$ the azimuth. Assume that the surface at $y(x \leqslant y \leqslant z)$ within the atmosphere is an intersection surface. In other words, the atmosphere is assumed to consist of two adjacent layers bounded by surfaces at $x, y$ and $z$. Furthermore, assume that the emergent intensities are linearly related to the intensities of radiation incident on the boundaries. The present linearity does not hold in the case of diffuse reflection and transmission of polychromatic radiation by a slab.

Let the reflection and transmission operators in the layer bounded by surfaces at $x$ and $y$ be denoted by $r(x, y)[$ or $r(y, x)]$ and $t(x, y)[$ or $t(y, x)]$, because of the polarity of the optical properties of the media (cf. Ueno, 1960), where the operator $r(x, y)$ and $t(x, y)$ are related to the incident intensity $\mathbf{I}(x,-\boldsymbol{\Omega})$, and the operators $r(y, x)$ and $l(y, x)$ are related to the incident intensity 
$\mathbf{I}(y, \mathbf{\Omega})$. Similarly, let the reflection and transmission operators in the layer bounded by surfaces at $y$ and $z$ be denoted by $\rho(y, z)[$ or $\rho(z, y)]$ and $\tau(y, z)[$ or $\tau(z, y)]$.

Allowing for the above boundary conditions of the composite layers, the emergent intensities of radiation from the sublayers take the form:

$$
\begin{aligned}
\mathbf{I}(x, \mathbf{\Omega}) & =r(x, y) \mathbf{I}(x,-\mathbf{\Omega})+t(y, x) \mathbf{I}(y, \mathbf{\Omega}) \\
\mathbf{I}(y,-\mathbf{\Omega}) & =r(y, x) \mathbf{I}(y, \mathbf{\Omega})+t(x, y) \mathbf{I}(x,-\mathbf{\Omega}) \\
\mathbf{I}(y, \mathbf{\Omega}) & =\rho(y, z) \mathbf{I}(y,-\mathbf{\Omega})+\tau(z, y) \mathbf{I}(z, \mathbf{\Omega}) \\
\mathbf{I}(z,-\mathbf{\Omega}) & =\rho(z, y) \mathbf{I}(z, \mathbf{\Omega})+\tau(y, z) \mathbf{I}(y,-\mathbf{\Omega}) .
\end{aligned}
$$

Or more concisely,

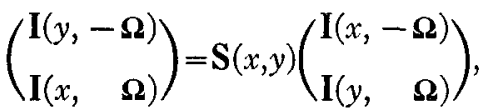

$$
\begin{aligned}
& \left(\begin{array}{rr}
\mathbf{I}(z, & -\mathbf{\Omega}) \\
\mathbf{I}(y, & \mathbf{\Omega})
\end{array}\right)=\mathbf{S}(y, z)\left(\begin{array}{cc}
\mathbf{I}(y, & -\mathbf{\Omega}) \\
\mathbf{I}(z, & \mathbf{\Omega})
\end{array}\right),
\end{aligned}
$$

$$
\mathbf{S}(x, z)=\left(\begin{array}{c}
\tau(y, z)[E-r(y, x) \rho(y, z)]^{-1} t(x, y) \\
r(x, y)+t(y, x) \rho(y, z)[E-r(y, x) \rho(y, z)]^{-1} l(x, y)
\end{array}\right.
$$

where $E$ represents the identity operator. It is of interest to mention that the star product requests the existence of the inverse operators $[E-r(y, x) \rho(y, z)]^{-1}$ or $[E-\rho(y, z) r(y, x)]^{-1}$. From the analytical and physical aspects, the inverse operators are expanded in a power series. For example,

$$
t(y, x) \rho(y, z) \sum_{i=0}^{\infty}[r(y, x) \rho(y, z)]^{i} t(x, y)
$$

denotes transmission through the upper layer bounded by the surfaces at $x$ and $y, i$ pairs of reflection back and forth at the interface, a final reflection from the lower layer bounded by the surfaces at $y$ and $z$, and transmission back through the upper layer. Thus, the required total reflection operator $\tau(x, z)$ consists of the reflection operator by the upper layer and the composite operator due to the sum of interactions involving reflection of all orders $n=0,1,2, \cdots$. It should be mentioned that the transmission integral operator consists of the directly transmitted component and the diffusely transmitted component.

Further, an alternative expression of the star product is obtained by using the following operator identities concerning the inverse operators:

$$
\begin{aligned}
(E-q p)^{-1} q & =q(E-p q)^{-1}, \\
(E-q p)^{-1} & =E+q(E-p q)^{-1} p,
\end{aligned}
$$

where $p$ and $q$ represent the integral operators.

$$
\left.\begin{array}{c}
\rho(z, y)+\tau(y, z) r(y, x)[E-\rho(y, z) r(y, x)]^{-1} \tau(z, y) \\
t(y, x)[E-\rho(y, z) r(y, x)]^{-1} \tau(z, y)
\end{array}\right),
$$

where

$$
\begin{aligned}
& \mathbf{S}(x, y)=\left(\begin{array}{ll}
l(x, y) & r(y, x) \\
r(x, y) & t(y, x)
\end{array}\right), \\
& \mathbf{S}(y, x)=\left(\begin{array}{ll}
\tau(y, z) & \rho(z, y) \\
\rho(y, z) & \tau(z, y)
\end{array}\right) .
\end{aligned}
$$

Taking into account the interactions of radiation at an arbitrary interface at $y$, the above expressions reduce to

$$
\left(\begin{array}{rr}
\mathbf{I}(z, & -\mathbf{\Omega}) \\
\mathbf{I}(x, & \mathbf{\Omega})
\end{array}\right)=\mathbf{S}(x, z)\left(\begin{array}{rr}
\mathbf{I}(x, & -\mathbf{\Omega}) \\
\mathbf{I}(z, & \mathbf{\Omega})
\end{array}\right) .
$$

It is readily shown that the elimination of $\mathbf{I}(y, \pm \mathbf{\Omega})$ from Eqs. (1)-(4) gives rise to Eq. (9). According to the terminology of Redheffer (1962), the star product of two two-by-two matrices is defined by

where

$$
\mathbf{S}(x, z)=\mathbf{S}(x, y) * \mathbf{S}(y, z),
$$

It is of interest to mention the analytical character of the star product:

(i) In general, the star product is non-commutative,

(ii) The star product is associative.

(iii) There exists an identity operator for the star product, namely, the scattering matrix for a layer of zero optical thickness:

$$
\mathbf{M}(x, x)=\left(\begin{array}{ll}
E & 0 \\
0 & E
\end{array}\right) .
$$

We can readily extend our present discussion to multiply-stratified layers.

\section{The doubling equations}

In a manner similar to that given in the previous section, we consider an inhomogeneous, anisotropically scattering atmosphere consisting of two component homogeneous layers of optical thicknesses $(y-x)$ and $(z-y)$. It is assumed that the radiation incident on the bottom from below vanishes. The intensities of radiation emergent from the top at $x$ and from the bottom at $z$ are given by Eq. (9), where

$$
\left.\begin{array}{ll}
r(x, y)=r(y, x), & l(x, y)=t(y, x) \\
\rho(y, z)=\rho(z, y), & \tau(y, z)=\tau(z, y)
\end{array}\right\},
$$

because of the non-polarity in the upper and lower layers. 
The reflection and transmission integral operators are defined by (Ueno and Wang, 1972)

$$
\begin{array}{r}
r(x, y)\left\{f\left(\mathbf{\Omega}^{\prime}\right)\right\}=\frac{1}{4 \pi v} \int_{2 \pi} \mathbf{S}\left(y-x ; \mathbf{\Omega}, \boldsymbol{\Omega}^{\prime}\right) f\left(\mathbf{\Omega}^{\prime}\right) d \Omega^{\prime}, \\
t(x, y)\left\{f\left(\mathbf{\Omega}^{\prime}\right)\right\}=\frac{1}{4 \pi v} \int_{2 \pi}\left[4 \pi v e^{-(y-x) / v^{\prime}} \delta\left(\mathbf{\Omega}-\mathbf{\Omega}^{\prime}\right)\right. \\
\left.\quad+\mathbf{T}\left(y-x ; \mathbf{\Omega}, \mathbf{\Omega}^{\prime}\right)\right] f\left(\mathbf{\Omega}^{\prime}\right) d \Omega^{\prime},
\end{array}
$$

where the $S$ - and $T$-functions are called the scattering and transmission functions, $d \Omega^{\prime}=d v^{\prime} d \varphi^{\prime}$, and the $\delta$ function is the Dirac delta function given by

$$
\delta\left(\Omega-\Omega^{\prime}\right)=\delta\left(v-v^{\prime}\right) \delta\left(\varphi-\varphi^{\prime}\right) .
$$

The integration interval with respect to $\Omega^{\prime}$ covers the range $(0,1)$ with respect to $v^{\prime}$ and the range $(0,2 \pi)$ with respect to $\varphi^{\prime}$.

The intensities of diffusely reflected and transmitted radiation by the atmosphere are expressed in the form

$$
\begin{aligned}
\mathbf{I}(x, \boldsymbol{\Omega}) & =\frac{F}{4 v} \mathbf{S}\left(z-x ; \boldsymbol{\Omega}, \boldsymbol{\Omega}_{0}\right), \\
\mathbf{I}(z,-\boldsymbol{\Omega}) & =\frac{F}{4 v} \mathbf{T}\left(z-x ; \boldsymbol{\Omega}, \boldsymbol{\Omega}_{0}\right),
\end{aligned}
$$

where $\boldsymbol{\Omega}_{0}$ stands for $\left(u, \varphi_{0}\right)$, and the flux $\pi F$ of radiation per unit area normal to itself is incident on the top.

Similarly, we can define such scattering and transmission functions for the lower layer as $\mathbf{S}\left(z-y ; \boldsymbol{\Omega}, \boldsymbol{\Omega}_{0}\right)$ and $\mathbf{T}\left(z-y ; \boldsymbol{\Omega}, \boldsymbol{\Omega}_{0}\right)$. With the aid of Eq. (9), we determine the intensity of radiation emergent from the whole atmosphere.

In what follows, we shall find the doubling equations of radiation, if the optical properties of the turbid media are constant throughout the whole atmosphere. Furthermore, we assume that the optical thickness of the upper layer coincides with that of the lower layer, i.e., $z-y=y-x=l$. We then have

$$
\begin{aligned}
& \mathbf{S}\left(l ; \boldsymbol{\Omega}, \boldsymbol{\Omega}_{0}\right)=\mathbf{S}\left(y-x ; \boldsymbol{\Omega}, \boldsymbol{\Omega}_{0}\right)=\mathbf{S}\left(z-y ; \boldsymbol{\Omega}, \boldsymbol{\Omega}_{0}\right), \\
& \mathbf{T}\left(l ; \boldsymbol{\Omega}, \boldsymbol{\Omega}_{0}\right)=\mathbf{T}\left(y-x ; \boldsymbol{\Omega}, \boldsymbol{\Omega}_{0}\right)=\mathbf{T}\left(z-y ; \boldsymbol{\Omega}, \boldsymbol{\Omega}_{0}\right) .
\end{aligned}
$$

Starting with Eq. (9) together with Eq. (11), defining

$$
\mathbf{I}(x,-\mathbf{\Omega})=\pi F \mathbf{\delta}\left(\mathbf{\Omega}-\mathbf{\Omega}_{0}\right) \text {, }
$$

recalling Eqs. (15), (16), (17), (21) and (22), and expanding the inverse operators into the power series, after minor rearrangements of some terms, we get

$$
\begin{aligned}
& \underset{4 \eta}{F} \mathbf{S}\left(2 l ; \boldsymbol{\Omega}, \mathbf{\Omega}_{0}\right) \\
& =\left[r(l)+l(l) \sum_{i=0}^{\infty} r(l)^{2 i+1} t(l)\right]\left\{\mathbf{I}\left(x,-\mathbf{\Omega}^{\prime}\right)\right\},
\end{aligned}
$$

where $r(l)=r(y, x)=r(z, y), t(l)=t(y, x)=t(z, y)$.
Introducing the direct transmission operator $t^{r}$ describing the reduced incident radiation and the diffuse transmission operator $l^{*}$ governing the diffuse radiation field, we obtain

$$
t(l)\left\{f\left(\mathbf{\Omega}^{\prime}\right)\right\}=\operatorname{lr}(l)\left\{f\left(\boldsymbol{\Omega}^{\prime}\right)\right\}+t^{*}(l)\left\{f\left(\boldsymbol{\Omega}^{\prime}\right)\right\}
$$

where

$$
\begin{aligned}
& l^{r}(l)\left\{f\left(\boldsymbol{\Omega}^{\prime}\right)\right\}=\int_{2 \pi} e^{-l l v^{\prime}} \delta\left(\boldsymbol{\Omega}-\boldsymbol{\Omega}^{\prime}\right) f\left(\boldsymbol{\Omega}^{\prime}\right) d \Omega^{\prime}, \\
& t^{*}(l)\left\{f\left(\boldsymbol{\Omega}^{\prime}\right)\right\}=\frac{1}{4 \pi v} \int_{2 \pi} \mathbf{T}\left(l ; \boldsymbol{\Omega}, \boldsymbol{\Omega}^{\prime}\right) f\left(\boldsymbol{\Omega}^{\prime}\right) d \Omega^{\prime} .
\end{aligned}
$$

From Eq. (24) we have

$$
\begin{aligned}
& \underset{4 v}{F} \mathbf{S}\left(2 l ; \boldsymbol{\Omega}, \boldsymbol{\Omega}_{0}\right)=\left[r(l)+t^{r}(l) \sum_{i=0}^{\infty} r(l)^{2 i+1} t^{r}(l)\right. \\
&+t^{*}(l) \sum_{i=0}^{\infty} r(l)^{2 i+1} t^{r}(l)+t^{r}(l) \sum_{i=0}^{\infty} r(l)^{2 i+1} t^{*}(l) \\
& \\
&\left.+t^{*}(l) \sum_{i=0}^{\infty} r(l)^{2 i+1} t^{*}(l)\right]\left\{\mathbf{I}\left(x,-\mathbf{\Omega}^{\prime}\right)\right\}
\end{aligned}
$$

After some minor manipulations, Eq. (28) reduces to

$\mathbf{S}\left(2 l ; \boldsymbol{\Omega}, \boldsymbol{\Omega}_{0}\right)=\mathbf{S}\left(l ; \boldsymbol{\Omega}, \boldsymbol{\Omega}_{0}\right)+\mathbf{\Sigma}_{0}\left(l ; \boldsymbol{\Omega}, \boldsymbol{\Omega}_{0}\right) e^{-x(1 / v+1 / u)}$

$$
\begin{array}{r}
+\frac{1}{4 \pi} e^{-x / u} \int_{2 \pi} \mathbf{T}\left(l ; \boldsymbol{\Omega}, \boldsymbol{\Omega}^{\prime}\right) \mathbf{\Sigma}_{0}\left(l ; \boldsymbol{\Omega}^{\prime}, \boldsymbol{\Omega}_{0}\right) \frac{d \Omega^{\prime}}{v^{\prime}} \\
+\frac{1}{4 \pi} e^{-x / v} \int_{2 \pi} \mathbf{\Sigma}_{0}\left(l ; \boldsymbol{\Omega}, \boldsymbol{\Omega}^{\prime}\right) \mathbf{T}\left(l ; \boldsymbol{\Omega}^{\prime}, \boldsymbol{\Omega}_{0}\right) \frac{d \Omega^{\prime}}{v^{\prime}} \\
+\frac{1}{(4 \pi)^{2}} \int_{2 \pi} \int_{2 \pi} \mathbf{T}\left(l ; \boldsymbol{\Omega}, \boldsymbol{\Omega}^{\prime}\right) \mathbf{\Sigma}_{0}\left(l ; \boldsymbol{\Omega}^{\prime}, \boldsymbol{\Omega}^{\prime \prime}\right) \\
\times \mathbf{T}\left(l ; \boldsymbol{\Omega}^{\prime \prime}, \boldsymbol{\Omega}_{0}\right) \frac{d \Omega^{\prime}}{v^{\prime}} \frac{d \Omega^{\prime \prime}}{v^{\prime \prime}}
\end{array}
$$

where

$$
\begin{aligned}
& \mathbf{\Sigma}_{0}\left(l ; \boldsymbol{\Omega}, \boldsymbol{\Omega}_{0}\right)=\sum_{n=1,3, \ldots}^{\infty} \mathbf{S}_{n}\left(l ; \boldsymbol{\Omega}, \boldsymbol{\Omega}_{0}\right), \\
& \mathbf{S}_{1}\left(l ; \boldsymbol{\Omega}, \boldsymbol{\Omega}_{0}\right)=\mathbf{S}\left(l ; \boldsymbol{\Omega}, \boldsymbol{\Omega}_{0}\right), \\
& \mathbf{S}_{n}\left(l ; \boldsymbol{\Omega}, \boldsymbol{\Omega}_{0}\right)=\frac{1}{4 \pi} \int_{2 \pi} \mathbf{S}\left(l ; \boldsymbol{\Omega}, \boldsymbol{\Omega}^{\prime}\right) \mathbf{S}_{n-1}\left(l ; \boldsymbol{\Omega}^{\prime}, \boldsymbol{\Omega}_{0}\right) \frac{d \boldsymbol{\Omega}^{\prime}}{v^{\prime}} .
\end{aligned}
$$

Eq. (29) is the required doubling equation for the scattering function in a finite atmosphere.

Similarly, starting with Eq. (9), and recalling Eqs. (11), (15), (16), (17), (20), (21), (22), (23) and (25), 
we have

$$
\begin{aligned}
& \pi F e^{-2 l / \cdot \boldsymbol{\delta}}\left(\mathbf{\Omega}-\mathbf{\Omega}_{0}\right)+\frac{F}{4 v} \mathbf{T}\left(2 l ; \mathbf{\Omega}, \mathbf{\Omega}_{0}\right) \\
& =\left[t(l) \sum_{i=0}^{\infty} r(l)^{2 i} t(l)\right]\left\{\mathbf{I}\left(x,-\mathbf{\Omega}^{\prime}\right)\right\} \\
& =\left[t^{r}(l) \sum_{i=0}^{\infty} r(l)^{2 i} t^{r}(l)+t^{*}(l) \sum_{i=0}^{\infty} r(l)^{2 i} t^{r}(l)\right. \\
& \left.+\operatorname{rr}(l) \sum_{i=1}^{\infty} r(l)^{2 i} l^{*}(l)+l^{*}(l) \sum_{i=1}^{\infty} r(l)^{2 i} t^{*}(l)\right] \\
& \times\left\{\mathbf{I}\left(x,-\mathbf{\Omega}^{\prime}\right)\right\} .
\end{aligned}
$$

After some manipulations, the required doubling equation for the transmission function becomes

$$
\begin{aligned}
& \mathbf{T}\left(2 l ; \boldsymbol{\Omega}, \mathbf{\Omega}_{0}\right)=\mathbf{T}\left(l ; \boldsymbol{\Omega}, \boldsymbol{\Omega}_{0}\right)\left(e^{-l / u}+e^{-l / p}\right) \\
& +\mathbf{\Sigma}_{\boldsymbol{\epsilon}}\left(l ; \Omega, \mathbf{\Omega}_{0}\right) e^{-l(1 / u+1 / v)} \\
& +\frac{1}{4 \pi} \int_{2 \pi} \mathbf{T}\left(l ; \mathbf{\Omega}, \mathbf{\Omega}^{\prime}\right) \mathbf{T}\left(l ; \mathbf{\Omega}^{\prime}, \mathbf{\Omega}_{0}\right) \frac{d \Omega^{\prime}}{v^{\prime}} \\
& +\frac{1}{4 \pi} e^{-x / u} \int_{2 \pi} \mathbf{T}\left(l ; \mathbf{\Omega}, \mathbf{\Omega}^{\prime}\right) \mathbf{\Sigma}_{\epsilon}\left(l ; \mathbf{\Omega}^{\prime}, \mathbf{\Omega}_{0}\right) \frac{d \Omega^{\prime}}{v^{\prime}} \\
& +\frac{1}{4 \pi} e^{-x / v} \int_{2 \pi} \mathbf{\Sigma}_{\epsilon}\left(l ; \mathbf{\Omega}, \mathbf{\Omega}^{\prime}\right) \mathbf{T}\left(l ; \mathbf{\Omega}^{\prime}, \mathbf{\Omega}_{0}\right) \frac{d \Omega^{\prime}}{v^{\prime}} \\
& +\frac{1}{(4 \pi)^{2}} \int_{2 \pi} \int_{2 \pi} \mathbf{T}\left(l ; \boldsymbol{\Omega}, \mathbf{\Omega}^{\prime}\right) \mathbf{\Sigma}_{\epsilon}\left(l ; \mathbf{\Omega}^{\prime}, \boldsymbol{\Omega}^{\prime \prime}\right) \\
& \times \mathbf{T}\left(l ; \mathbf{\Omega}^{\prime \prime}, \mathbf{\Omega}_{0}\right) \frac{d \Omega^{\prime}}{v^{\prime}} \frac{d \Omega^{\prime \prime}}{v^{\prime \prime}},
\end{aligned}
$$

where

$$
\mathbf{\Sigma}_{\boldsymbol{\epsilon}}\left(l ; \boldsymbol{\Omega}, \mathbf{\Omega}_{0}\right)=\sum_{n=2,4}^{\infty} \mathbf{S}_{n}\left(l ; \boldsymbol{\Omega}, \mathbf{\Omega}_{0}\right) .
$$

The doubling equations given by Eqs. (29) and (34) reduce to those given by van de Hulst (1963) and Hansen (1969). For very thin layers the scattering and transmission functions of singly scattered radiation in terms of the given phase function by Eqs. (29) and (33) are equal to the first-order scattering and transmission functions in the order-of-scattering theory of radiation (cf. Bellman et al., 1972).

Finally, it should be mentioned that a complete set of invariance principles for the diffuse reflection and transmission problem (cf., Chandrasekhar, 1960; Eqs. (5)-(8), p. 162) is readily found either from Eqs. (1)-(4) or from Eqs. (5) and (6), recalling Eqs. (16), (17) and (25).

\section{REFERENCES}

Ambarzumian, V. A., Ed., 1958: Theoretical A strophysics. London, Pergamon Press.

Aronson, R., and D. L. Yarmush, 1966: Transfer-matrix method for gamma-rays and neutron penetration. J. Math. Phys., 7, 221-237.

Bellman, R. E., R. E. Kalaba and M. C. Prestrnd, 1963 : Invariant Imbedding and Radiative Transfer in Slabs of Finite Thickness. New York, Elsevier.

-_., S. Ueno and R. Vasudevan, 1972: Invariant imbedding and radiation dosimetry: II, Integral recurrence relations for the finite order scattering and transmission functions. Math. Bioscience, 15, 153-162.

Busbridge, I. W., 1960: The Mathematics of Radiative Transfer. Cambridge Tracts, No. 50, Cambridge University Press.

Chandrasekhar, S. 1960: Radiative Transfer. New York, Dover Publ.

Grant, I. P., and G. E. Hunt, 1969: Discrete space theory of radiative transfer I. Fundamentals. Proc. Roy. Soc. London, A313, 183-197.

Hansen, J. E., 1969: Radiative transfer by doubling very thin layers. Astrophys. J., 155, 565-573.

-_- and J. W. Hovenier, 1971: The doubling method applied to multiple scattering of polarized light. J. Quant. Spectros. Radial. Transfer, 11, 809-812.

Hovenier, J. W., 1971: Multiple scattering of polarized light in planetary atmospheres. Astron. Astrophys., 13, 7-29.

Peebles, G. H., and M. S. Plesset, 1951: Transmission of gammarays through large thickness of heavy materials. Phys. Rer', 81, 430-439.

Redheffer, R., 1962: On the reflection of transmission line theory to scattering and transfer. J. Math. Phys., 12,1-41.

Sobolev, V. V., 1962: A Treatise on Radiative Transfer. Princeton, N. J., Van Nostrand.

Ueno, S., 1960: The probabilistic method for problems of radiative transfer X. Diffuse reflection and transmission in a finite homogeneous atmosphere. Astrophys. J., 132, 729-745.

, and A. P. Wang, 1973 : Scattering and transmission functions of radiation by finite atmospheres with reflecting surfaces. Astropliys. Space Sci. (in press).

Uesugi, A., and W. M. Irvine, 1969: Computations of synthetic spectra for semi-infinite atmosphere. I. Almos. Sci., 26, 973-978.

Van de Hulst, H. C., 1963: A new look at multiple scattering. New York, NASA Institute for Space Studies.

Wang, A. P. 1966: Scattering process. Ph.D. thesis, University of California, Los Angeles. 